\title{
Kidney Transplant Recipient with Conversion Disorder Treated by Electroconvulsive Therapy
}

\author{
Rachel Beeson ${ }^{a}$ Stavros Stefanopoulos ${ }^{a, b}$ Daniel Rapport ${ }^{1, c}$ and Jorge Ortiz ${ }^{a}$
}

Coresponding author(s): daniel.rapport@utoledo.edu

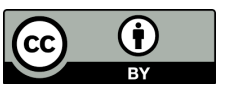

\begin{abstract}
${ }^{a}$ Department of Surgery The University of Toledo, Health Science Campus, 3000 Arlington Ave., Toledo, OH 43614, USA, ${ }^{b}$ M.D. Candidate, Class of 20XX, The University of Toledo Heath Science Campus, 3000 Arlington Ave., Toledo, OH 43614, USA, and ${ }^{c}$ Department of Psychiatry, The University of Toledo, Health Science Campus, 3000 Arlington Ave., Toledo, $\mathrm{OH} 43614$, USA
\end{abstract}

\begin{abstract}
We describe the case of a patient with severe post-operative conversion disorder successfully treated with electroconvulsive therapy (ECT) with good clinical outcome. A 66-year-old Caucasian male presented to the emergency department (ED) with altered mental status and was nonverbal three days after undergoing an uncomplicated incisional hernia repair. He had a past medical history of major depression with psychotic features and generalized anxiety disorder as well as a kidney transplant. He had multiple previous psychiatric hospitalizations in the 1980 s for severe depression with suicidality treated successfully with ECT. The patient was admitted, and a diagnosis of conversion disorder was made. His condition deteriorated over 21 days of inpatient management and he failed to respond to methylphenidate, aripiprazole, haloperidol, and lorazepam. Bilateral ECT treatment was initiated and the patient demonstrated a dramatic functional improvement after the first treatment. He was discharged home after receiving 6 total treatments and continued outpatient treatments with good clinical outcome. ECT may be considered in patients with severe conversion disorder with a previous history of successful treatment.
\end{abstract}

conversion disorder | electroconvulsive therapy | kidney transplant

or millennia, conversion disorder has been a well-documented phenomenon troubling medical professionals and accruing various names over time, most notably hysteria $(1,2)$. Freud and Janet's work independently laid the foundation for the current psychological understanding we have of the disease today $(1,3-5)$. Today, the Diagnostic and Statistical Manual of Mental Disorders (DSM5) defines it as a psychiatric illness with symptoms affecting voluntary motor or sensory function that cannot be explained by an organic medical condition and cause clinically significant impairment in function (6). Making the diagnosis can present a challenge to medical professionals. Other neurologic or psychiatric conditions must be considered and ruled out including, non-epileptic seizures, factitious disorder, malingering, and somatization to name a few (3,
7). The current treatment for conversion disorder is psychotherapy as the basis, but can also include physical therapy and medications such as SSRIs (3, 7-9). ECT is seldom used in the treatment of conversion disorder, however this treatment has been demonstrated in multiple case reports and case studies to have been beneficial in refractory patients (10-13). We present the case of a patient exhibiting severe conversion disorder treated with ECT resulting in good clinical outcome.

\section{Case Report}

Patient Information

A 66-year-old Caucasian male presented to the emergency department (ED) with altered mental status 3 days after undergoing an uncomplicated incisional hernia repair. The patient had become nonverbal at home and was brought to the ED by his family for evaluation. The family further elaborated on the abnormal behavior claiming the patient had signs of paranoia, hallucinations, and disorganized speech. The patient had a past psychiatric history significant for major depression with psychotic features and generalized anxiety disorder. He had a previous history of psychiatric hospitalizations in the 1980s for severe depression with suicidality which were successfully treated with ECT. He was subsequently maintained on lithium carbonate to control his psychiatric disorder. The lithium treatment was complicated by renal failure and he received a renal transplant in 2016 from a related living donor. He was maintained on anti-rejection medications thereafter. Following the renal transplant, he was placed on aripiprazole to prevent further relapse of his psychiatric condition. His family had not observed any other

Submitted: 01/17/202, published: 05/14/2020 
signs of abnormal behavior, depression, or withdrawal in the weeks leading up to the hernia repair. However, they did report that he had been anxious about the surgery and stated that he had had a similar, albeit less severe, reaction following his renal transplant. On exam in the emergency department, the patient was alert but difficult to assess given his nonverbal condition. He responded to painful stimuli with facial grimacing. Observation of the surgical site was unremarkable and he was afebrile. His vital signs, complete blood count, complete metabolic panel, troponins, and creatinine kinase were all within normal limits. Toxicology screen was negative. A CT scan of the abdomen, pelvis, and brain did not reveal any acute abnormalities.

\section{Objective for Case Reporting}

To present a novel case of a patient with severe conversion disorder treated with ECT with good clinical outcome.

\section{Case}

The patient was admitted to the inpatient psychiatric unit for further evaluation and treatment. Aripiprazole was discontinued and switched to haloperidol empirically because it was felt to be a more potent antipsychotic than the low dose of aripiprazole that he was receiving. He continued to decline. Subsequently he refused any oral intake and by the third day he became bedbound and immobile. Given his presentation, a diagnosis of catatonia was now entertained considering his history of major depression with psychotic features. However, we could not establish the diagnosis of depression since the patient was mute. Additionally, he failed to respond to or show any improvement after receiving a $2 \mathrm{mg}$ dose of IV lorazepam.

After 3 days of management in the adult psychiatric inpatient service, he was transferred to the medicine unit under the transplant service and consultation liaison psychiatry was called in. A neurology consult was placed, and EEG was performed which ruled out status epilepticus. An MRI of the brain was unremarkable for acute pathology. Haloperidol was discontinued and low dose aripiprazole was restarted. Switching antipsychotics resulted in minimal improvement in the patient's state, although the family reported that he responded to them at times. This was viewed by the consult service as being inconsistent with typical signs of catatonia. He squeezed his wife's hand when prompted and was able to sing simple songs on command including happy birthday.

Aside from these isolated events, the patient remained stuporous, immobile, and nonverbal. There was no response to sternal rubs and no demonstration of rigidity, posturing, or any other notable mannerisms of catatonia. He did not exhibit negativism, opposition, or inability to respond to instructions. At this point, both his white blood cell count and body temperature were beginning to rise. Ultimately, the decision to consider ECT was necessary to prevent further morbidity and potential mortality. However, both the surgeon and consultation liaison psychiatrist agreed that the patient lacked capacity. The consultation liaison psychiatrist called in a second psychiatrist specializing in ECT who concurred. Before resorting to ECT, a brief trial of low-dose methylphenidate was given in the hopes that it might activate the patient and was gradually increased over 2 days. Shortly thereafter, the family reported that he appeared more anxious and uncomfortable at which point that was discontinued. After 3 weeks in the hospital with no improvement and increasing complications the risks, benefits, and rationale for ECT were discussed at length with the patient's wife and family. Given his history of responding positively to ECT in the past for major depression with psychotic features, the family agreed. The physician on the primary service concurred and the psychiatrist specializing in ECT treatment agreed. Consideration was given to obtaining a court order but further delay in treatment was considered hazardous. Bilateral ECT treatment was initiated and the patient demonstrated a dramatic functional improvement after the first treatment. However, this response was not sustained. Subsequent treatments ultimately resulted in marked and sustained improvement to the point where the patient was able to begin eating, sitting up at the bedside, ambulating and improvement ultimately leading to discharge from the hospital. He was discharged home after receiving 6 total treatments with plans for additional outpatient treatment.

At follow-up several months after his final ECT treatment he denied any anxiety, depression or symptoms of psychosis. He had fully returned to his baseline level of functioning with no residual sequelae from his hospitalization or the treatments.

\section{Discussion}

Considering the patients recent intra-abdominal surgery, a broad differential was considered including delirium secondary to uremia, infections, metabolic derangements, or intestinal incarceration. However, he was afebrile and his medical workup was consistently negative. His antirejection medications were considered as a potential contributor to his condition. Tacrolimus has been noted to cause psychosis and mycophenolate has been associated with increased anxiety $(14,15)$. However, he had been on these medications since his transplant in 2016 and not experienced these adverse effects.

Catatonia secondary to major depression with psychotic features was the initial working diagnosis but discrepancies in his presentation brought put this in doubt. He also failed to respond to one dose of $2 \mathrm{mg}$ of IV lorazepam. Though a full lorazepam challenge test involves a second dose if the patient does not respond, most catatonic patients respond within 10 minutes; our patients lack of response made catatonia a less likely diagnosis (16). Given the patient had absolutely no response to the first lorazepam dose, it was decided not continue with a second. Benzodiazepines are considered to be the Gold standard for confirming a diagnosis of catatonia as most cases will respond with a marked reduction of symptoms within 10 minutes of a 2-4 mg IV lorazepam (17). A "lorazepam test" cannot only confirm the diagnosis of catatonia but can also bring the underlying psychopathology to light by enabling patients to speak (18).

Conversion disorder, factitious disorder and malingering were also considered but the latter two were ruled out. The patient had been known to the department giving his recent surgery and treatment at our hospital. He had never shown previous symptoms of malingering or factitious disorder. His past psychiatric history was also considered and made these diagnoses much less likely. The clinical spectrum of conversion disorder is wide ranging. According to DSM 5, conversion disorder must have one or more symptoms of altered voluntary motor or sensory function with clinical findings that are incompatible with recognized neurological or medical conditions (6). This patient not only met these criteria, but he also experienced significant psychologic and physical stress as a result of his surgical procedure, providing a plausible explanation for his conversion symptoms. He had also expressed foreboding prior to the procedure. Additionally, it is common for patients with conversion disorder to suffer an underlying major depressive disorder. It was not inconceivable that this was the case.

There have been a number of postoperative conversion disorder cases reported in the literature (19-21). Furthermore, there have been multiple documentations of conversion disorder presenting 
with catatonia-like features similar to this patient's $(22,23)$. The treatment of the mute unresponsive patient requires a multidisciplinary approach with a combination of therapies. However, there are scarce controlled studies of the treatment of such patients and most information regarding the effectiveness of particular interventions is anecdotal (24). Psychotherapy, either psychodynamic or cognitive behavioral, continues to be the standard of care for conversion disorder $(3,7)$. However, given the state of the patient, that was not a plausible option. There are very few trials exploring the use of pharmacotherapy, one study showing modest improvements with SSRIs (9). Conversion disorder is not currently accepted as an indication for the use of ECT. However, ECT has been demonstrated in multiple cases to have been beneficial in patients with severe conversion disorder (10-12). Other studies have shown a favorable response to ECT treatment for various somatoform disorders, particularly cases with comorbid mood disorders (13). Although the exact mechanism of ECT is unknown, its efficacy in treating mood and thought disorders is well established (25). Major depression with psychotic features can be a common underlying etiology of both catatonia and conversion disorder and therefore may respond

1. Nicholson, T., J. Stone, and R. Kanaan,(2011), Conversion disorder: a problematic diagnosis. J Neurol Neurosurg Psychiatry, 82: p. 1267-1273.

2. Veith, I., Hysteria: the history of a disease. (1993), Northvale, New Jersey: Jason Aronson.

3. Ali, S., et al., (2015), Conversion Disorder| Mind versus Body: A Review. Clin Neurosci, 12(5-6): p. 27-33.

4. Freud, S., J. Strachey, and A. Freud, (1953), The standard edition of the complete psychological works of Sigmund Freud, London: Hogarth Press.

5. Janet, P., (1907), The major symptoms of hysteria: fifteen lectures given in the Medical School of Harvard University, New York: Macmillan.

6. Association AP. (2013), Diagnostic and statistical manual of mental disorders (DSM-5B). 5th ed. Washington, DC: American Psychiatric Pub. 318-321.

7. Stonnington, C., J. Barry, and R. Fisher, (2006), Conversion Disorder. Am J Psychiatry, 2006. 163(9): p. 1510-1517.

8. Kaur, J., et al., (2012), Conversion Disorder and Physical Therapy. Delhi Psychiatry Journal, 15(2): p. 394-397.

9. Voon V and Lang AE. (2005), Antidepressant treatment outcomes of psychogenic movement disorder. The Journal of clinical psychiatry.

10. Giovanoli EJ. (1988), ECT in a Patient with Conversion Disorder. Convulsive therapy.

11. Gaillard A, Gaillard R, Mouaffak F, et al. (2012), Case report: electroconvulsive therapy in a 33-year-old man with hysterical quadriplegia. L'Encephale, 38(1):104109.

12. Daniel, W.F., R.A. Yeo, and J.E. Smith, (1989), Conversion Disorders and ECT. The British Journal of Psychiatry, 154(2): p. 274-275.

13. Leong K, Tham JC, Scamvougeras A, et al. (2015), Electroconvulsive therapy treatment in patients with somatic symptom and related disorders. Neuropsychiatric disease and treatment, 11:2565. to ECT, especially in an emergency.

\section{Conclusion}

ECT in the treatment of severe conversion disorder may be appropriate and result in good clinical outcome. This is an example and a reminder of how ECT may be a useful treatment in severe conversion disorder when other options have failed.

\section{Conflict of interest}

Authors declare no conflict of interest.

\section{Authors' contributions}

SS, RB, DR, JO wrote, edited manuscript and reviewed paper. All authors read and approved the final document.

14. Bourgeois, J.A. and A. Hategan, (2014), Immunosuppressant-associated neurotoxicity responding to olanzapine. Immunosuppressant-associated neurotoxicity responding to olanzapine, (250472).

15. Krishna, N., et al., 92013), Tacrolimus-induced paranoid delusions and fugue-like state. General Hospital Psychiatry, 35(3): p. 327e5-327e6.

16. Sienaert, P., et al., (2014), A clinical review of the treatment of catatonia. Front Psychiatry, 5: p. 181.

17. Wilcox JA and Reid Duffy P. (2015), The syndrome of catatonia. Behavioral Sciences, 5(4):576-588.

18. Fink M and Taylor MA. (2006), Catatonia: a clinician's guide to diagnosis and treatment. Cambridge University Press.

19. Nelson EJ and Wu JY. (2017), Postoperative conversion disorder presenting as inspiratory stridor and hemiparesis in a pediatric patient. The American journal of case reports, 18:60.

20. Haden R. (2004), Conversion reaction following anaesthesia. Anaesthesia, 59(7):728-729.

21. Judge $A$ and Spielman F. (2010), Postoperative conversion disorder in a pediatric patient. Pediatric Anesthesia, 20(11):1052-1054.

22. Wiener M and Pauline K. (1990), A Case of Conversion Catatonia Misdiagnosed for 24 Years. Jefferson Journal of Psychiatry, 8(1):10.

23. Shah JL, Meyer FL, Mufson MJ, et al. (2012), Catatonia, conversion, culture: an acute presentation. Harvard review of psychiatry, 20(3):160-169.

24. Rosebush PI and Mazurek MF. (2011), Treatment of conversion disorder in the 21st century: have we moved beyond the couch? Current treatment options in neurology, 13(3):255-266.

25. Schonfeldt-Lecuona C, Lefaucheur JP, Lepping P, et al. (2016), Non-Invasive Brain Stimulation in Conversion (Functional) Weakness and Paralysis: A Systematic Review and Future Perspectives. Front Neurosci, 10:140. 